\title{
High- $Q$ measurements of fused-silica microspheres in the near infrared
}

\author{
D. W. Vernooy, V. S. Ilchenko, ${ }^{*}$ H. Mabuchi, E. W. Streed, and H. J. Kimble \\ Norman Bridge Laboratory of Physics 12-33, California Institute of Technology, Pasadena, California 91125
}

Received November 11, 1997

\begin{abstract}
Measurements of the quality factor $Q \approx 8 \times 10^{9}$ are reported for the whispering-gallery modes (WGM's) of quartz microspheres for the wavelengths 670,780 , and $850 \mathrm{~nm}$; these results correspond to finesse $\mathcal{F} \approx 2.2 \times 10^{6}$. The observed independence of $Q$ from wavelength indicates that losses for the WGM's are dominated by a mechanism other than bulk absorption in fused silica in the near infrared. Data obtained by atomic force microscopy combined with a simple model for surface scattering suggest that $Q$ can be limited by residual surface inhomogeneities. Absorption by absorbed water can also explain why the material limit is not reached at longer wavelengths in the near infrared. (c) 1998 Optical Society of America

OCIS codes: $270.0270,230.5750,160.6030$.
\end{abstract}

Small optical resonators with large quality factors $Q$ have diverse technological and scientific applications, ranging from frequency stabilization of semiconductor diode lasers ${ }^{1}$ to environmental sensing by trace absorption detection in compact, integrated structures. ${ }^{2}$ Our own interest in such resonators arises within the realm of cavity QED, in which it is necessary to achieve both small-cavity mode volumes (for large electric fields per photon) and ultralow resonator losses (for long photon-storage times). ${ }^{3}$ In the optical domain these conditions have been met only in small Fabry-Perot cavities, most recently for single atoms coupled one by one to a cavity of mode volume $V \approx 2 \times 10^{5} \mu \mathrm{m}^{3}$ and finesse $\mathcal{F} \approx 2 \times 10^{5}$. An attractive alternative for achieving these conditions is the whispering-gallery modes (WGM's) of quartz microspheres, as was pointed out in the pioneering research of Ref. 5. These modes can have both large coupling coefficients for the atomcavity interaction (mode volume $V \approx 5 \times 10^{3} \mu \mathrm{m}^{3}$; Refs. 6-8) and extremely low losses. ${ }^{6,9,10}$ Moreover, measurements in Ref. 11 demonstrate that experimental $Q$ factors can reach the intrinsic absorption limit of fused silica [at $633 \mathrm{~nm}, Q=(8 \pm 1) \times 10^{9}$ was reported]. For use in cavity QED and elsewhere, the microsphere WGM's are thus worthy of immediate further investigation, especially with the possibility of even higher $Q$ factors in the near infrared (NIR), as the bulksilica absorption coefficient decreases more than fivefold as the wavelength moves from $600 \mathrm{~nm}$ to $1 \mu \mathrm{m}$.

In this Letter we report what we believe to be the highest $Q$ 's for WGM's that have been achieved to date for wavelengths in the NIR. In addition, a measurement of $Q=4.9 \times 10^{9}$ at $670 \mathrm{~nm}$ in a 345 - $\mu$ m-diameter sphere corresponds to what is to our knowledge the highest finesse $\left(\mathcal{F}=2.3 \times 10^{6}\right)$ yet recorded for an optical resonator. Furthermore, our data reveal that $Q$ is largely independent of wavelength, so that the bulk absorption limit demonstrated in Ref. 11 is not tracked into the NIR, and the correspondingly higher $Q$ values are not obtained. A systematic investigation of the surface of the microsphere by atomic force microscopy (AFM) allows the identification of residual surface inhomogeneities of size $\sigma \simeq 1.7 \mathrm{~nm}$, and a simple model of surface scattering supports both the magnitude of the measured $Q$ values and their dependence on sphere size. A second mechanism, namely, optical absorption by water adsorbed on the sphere's surface, can quantitatively explain why $Q$ is limited to the NIR above $\lambda \approx 700 \mathrm{~nm}$.

The spheres were fabricated by use of an oxygenhydrogen microtorch to melt the ends of low-oxygenhydrogen silica rods, as was done in Ref. 12. Nearly spherical balls of ellipticity of $\leqslant 3 \%$ were thus formed. For each of the wavelengths 670,780 , and $850 \mathrm{~nm}$, the excitation source was an external-grating-stabilized diode laser of a few hundred kilohertz' linewidth. The laser output was coupled into the microsphere by frustrated total internal reflection from a coupling prism, ${ }^{9}$ without contact between the sphere and the prism. To obtain a clean output beam, we injected the in-coupling beam at an angle to the sphere's equatorial plane to take advantage of the so-called precessing modes. ${ }^{13}$ Cavity lifetimes were determined by a ringdown measurement with the incident field gated off by an acousto-optic modulator.

Although mode splittings (as in Ref. 14) are seen in our work for $Q \geq 5 \times 10^{8}$, the particular results reported here have an observed resonator energy decay that is well fitted by $\exp -t / \tau=\exp (-\omega t / Q)$, which corresponds to excitation of predominantly one eigenmode. The highest $Q$ values inferred from the recorded decay times are $(8 \pm 1) \times 10^{9}$ at $670 \mathrm{~nm}$, $(7.5 \pm 1) \times 10^{9}$ at $780 \mathrm{~nm}$, and $(7.2 \pm 1) \times 10^{9}$ at $850 \mathrm{~nm}$ in spheres of diameter $D=750,800$, and $680 \mu \mathrm{m}$, respectively. These $Q$ values were reproduced in at least one other sphere for all three wavelengths. In Fig. 1 we plot our three measurements along with the point from Ref. 11 at $633 \mathrm{~nm}$ with $D=750 \mu \mathrm{m}$. Also shown in Fig. 1 is the expected variation for $Q$ if bulk material absorption is the limit [i.e., $Q_{\text {mat }}=n k / \alpha_{\text {mat }}$, with refractive index $n=1.452$, $k=2 \pi / \lambda$, and the absorption coefficient $\alpha_{\text {mat }}(\lambda)$ in very low-grade oxygen-hydrogen fiber-grade fused silica that was given in Ref. 15]. Although the two data points at 633 and $670 \mathrm{~nm}$ approach $Q_{\mathrm{abs}}$, the data at 780 and $850 \mathrm{~nm}$ in the NIR fall substantially short. One possible reason for reduced $Q$ is surface contamination, although our cleaning procedure eliminates vir- 


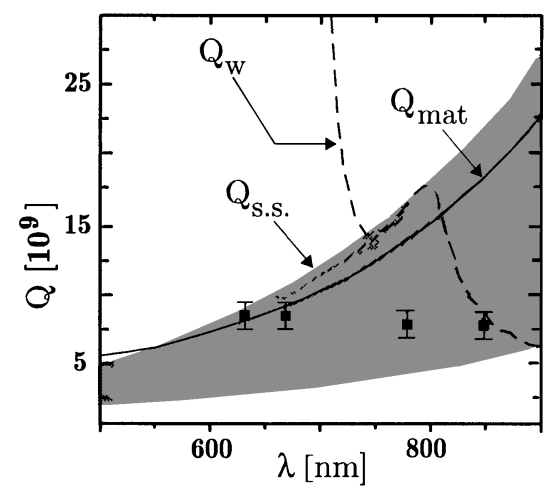

Fig. 1. The highest $Q$ values measured at 670,780 , and $850 \mathrm{~nm}$ are shown, along with the wavelength dependencies for $Q_{\text {mat }}$ limited by intrinsic material absorption (solid curve), $Q_{\mathrm{ss}}$ for surface scattering (the shaded region shows a bound with AFM surface data), and $Q_{w}$ for an adsorbed layer of water (dashed curve). The point at $633 \mathrm{~nm}$ is from Ref. 11.

tually all diffuse scattering from particles on the preform surface. Anecdotally, it is our experience that high $Q$ is never achieved when such scattering is seen. For the large spheres employed here, the limit set by radiative losses is irrelevant $\left(Q_{\mathrm{rad}}>10^{20}\right.$ for $D \gtrsim 500 \mu \mathrm{m})$.

We undertook a quantitative investigation of the role of scattering from surface inhomogeneities as another loss mechanism (related to the annealing history of the silica ball when it is withdrawn from the flame) and found AFM to be most fruitful in providing quantitative microsphere surface data. In Fig. 2 we present a surface-morphology scan taken over a $20 \mathrm{~nm} \times 20 \mathrm{~nm}$ grid $(256 \times 256$ points $)$ with a nominal vertical resolution of $0.01 \mathrm{~nm}$. By organizing these data as 256 line scans of 256 points each [i.e., heights $h_{i}\left(x_{j}\right)$, with $x_{j}=20 j / 256$ (in nanometers), $i, j=\{1 \ldots 256\}$, and $\left.\left\langle h_{i}\left(x_{j}\right)\right\rangle_{i, j}=0\right]$, we calculate an average standard deviation of surface roughness $\bar{\sigma}=(1.7 \pm 0.5) \mathrm{nm}$. In addition, for each line scan we calculate $R_{i}(u)=\left\langle h_{i}\left(x_{j}\right) h_{i}\left(x_{j}+u\right)\right\rangle_{j}$ and then construct an average correlation function $R(u)=\left\langle R_{i}(u)\right\rangle_{i}$, which is shown in Fig. 3(a). From this plot we identify a statistical correlation length $B=(5 \pm 0.5) \mathrm{nm}$. The Fourier transform $P(k)$ of $R(u)$ gives the power spectrum of surface fluctuations in reciprocal space, and an estimate for $P(k)$ in Fig. 3(b) is correspondingly flat out to a spatial frequency $k_{0} \sim 1 / B$.

Given $\sigma$ and $B$, the surface-scattering-limited $Q_{\mathrm{ss}}$ must take into account precisely how the energy of the resonant-mode field distribution is scattered into the complete set of modes pertinent to the spherical geometry. For example, surface-scattering limits are expected to depend on radial mode number $q$, but because the modes that are excited here have $q \lesssim 3$, a simple estimate can be based on scattering from particles of dielectric constant $\epsilon=n^{2}$ and size $\sigma B^{2} \ll$ $\lambda^{3}$. Using the volumetric ratio of a surface layer of such particles to that of the mode, $\sim \sigma / \sqrt{\lambda D}$, one finds that

$$
Q_{\mathrm{ss}} \sim \frac{3 \epsilon(\epsilon+2)^{2}}{(4 \pi)^{3}(\epsilon-1)^{5 / 2}} \frac{\lambda^{7 / 2} D^{1 / 2}}{\sigma^{2} B^{2}}
$$

We can derive an equivalent expression with a different approach based on surface scattering from planar waveguides ${ }^{16}$ by substituting the ratio of the external to the internal mode volumes of the sphere, $\sim \sqrt{\lambda / D}$, for the ratio of the external to the internal guided power in the waveguide. Using $\sigma$ and $B$ from above, $D=800 \mu \mathrm{m}$, and $\lambda=800 \mathrm{~nm}$, we estimate that $Q_{\mathrm{ss}} \sim(7.5 \pm 5) \times 10^{9}$, which compares well with our measurements $\left(\sim 8 \times 10^{9}\right)$.

In Fig. 4 we plot the highest $Q$ achieved as a function of $D$ for our experience with spheres at fixed $\lambda=$ $670 \mathrm{~nm}$, with the caveat that the repeatability in obtaining the highest $Q$ for a given $D$ accounts for the considerable scatter in the data. Nevertheless, a fit of $\propto D^{1 / 2}$ as suggested by relation (1) yields $\sigma B \sim$ $5 \mathrm{~nm}^{2}$, which coincides well with our analysis of the experimental AFM data. Although the trend in Fig. 4 argues for measurements with still-larger spheres, our fabrication technology is not suited for $D$ much in excess of $1 \mathrm{~mm}$.

As in Ref. 11, we find a time-dependent reduction in $Q$ (presumably owing to absorption by water on the surface), with the highest $Q$ data measured within $\Delta t=1.5 \mathrm{~min}$ of fabrication. We admit the real possibility that even higher $Q$ can be recorded in a dry environment or for $\Delta t \rightarrow 0$. In fact, for a water layer of width $\delta \ll \lambda / 2 \pi$ occupying a fraction of the total mode volume $\propto \delta / \sqrt{\lambda D}$ and absorption coefficient $\beta_{w}(\lambda)$, we estimate that

$$
Q_{w} \sim \sqrt{\frac{\pi}{8 n^{3}}} \frac{D^{1 / 2}}{\delta \lambda^{1 / 2} \beta_{w}(\lambda)},
$$

which shows the same $D^{1 / 2}$ dependence as $Q_{\mathrm{ss}}$. Although the exact chemistry of the surface-adsorption process would seem to be important in determining

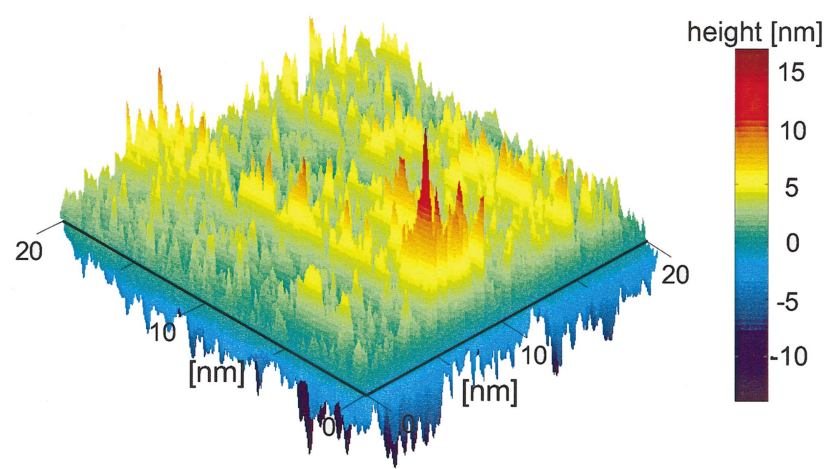

Fig. 2. Surface data obtained by AFM on a 20 -nm square grid of $256 \times 256$ points show a rms roughness $\sigma \sim 2 \mathrm{~nm}$.

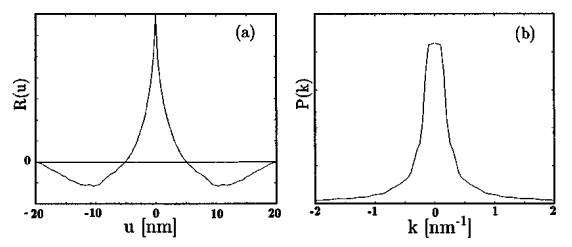

Fig. 3. (a) Correlation function $R(u)$ and (b) estimate of its Fourier transform, the power spectral density $P(k)$, shown after averaging over 256 scans on a $20-\mathrm{nm}$ square grid, as explained in the text. Both support the identification of the correlation length $B \sim 5 \mathrm{~nm}$. 


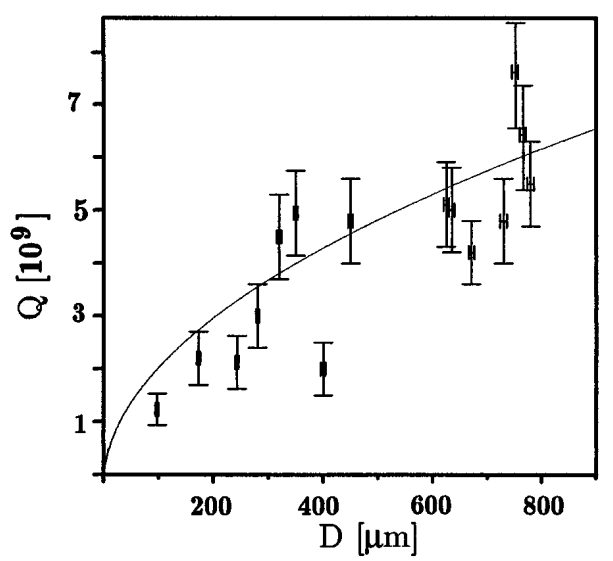

Fig. 4. The highest $Q$ values measured in spheres of diameter $D$ at $670 \mathrm{~nm}$. The dependence $Q_{\mathrm{ss}} \propto D^{1 / 2}$ (solid curve) is suggested by relation (1).

both $\beta_{w}(\lambda)$ and $\delta$ at the time $\Delta t$ of our measurements, an estimate with $\beta_{w}$ of bulk water in the $500-900-\mathrm{nm}$ range from Ref. 17 and $\delta \sim 0.2 \mathrm{~nm}$ as appropriate to one to two monolayers gives $Q_{w} \sim 7 \times 10^{10}$ for our data point at $670 \mathrm{~nm}$ with $D=750 \mu \mathrm{m}$ (likewise, the data at $670 \mathrm{~nm}$ in Fig. 4 would predict a value of $\delta$ roughly an order of magnitude too large). This estimate favors material absorption and surface-scattering losses at long visible wavelengths and small $\Delta t$. However, there is a rapid decrease to $Q_{w} \sim 8 \times 10^{9}$ at $850 \mathrm{~nm}$ (with $D=680 \mu \mathrm{m}$ ), strongly suggesting that water absorption plays a fundamental role farther into the NIR.

To summarize, we include in Fig. 1 the $\lambda$ dependence of $Q_{w}$ and $Q_{\mathrm{ss}}$ (using experimental limits for $\sigma$ and $B$ ) for a typical size $D \sim 700 \mu \mathrm{m}$, while cautioning that the data themselves have variable $D$. To remove uncertainty about the dominant loss mechanism at any given wavelength, one would like to inhibit hydration and so observe a clear shelf in $Q$ versus $\Delta t$ near $\Delta t=0$. Similarly, the dependence $Q_{\mathrm{ss}} \propto \lambda^{7 / 2}$, although apparently it is severe, changes by only a factor of $\sim 2.5$ from 650 to $850 \mathrm{~nm}$ and could be better identified by a larger dynamic range in $\lambda$. Alternatively, these effects might be mitigated with appropriate surface treatment. In any case, it is encouraging that spheres with $D \approx 100 \mu \mathrm{m}$ and $Q \approx 1 \times 10^{9}$ already yield critical atom and photon numbers $N_{0} \sim 10^{-3}$ and $m_{0} \sim 10^{-2}$ (Ref. 3) for cavity QED experiments.

Finally, we make contact with other measurements of resonator loss by use of the cavity finesse $\mathcal{F}$ for a free spectral range that corresponds to an additional wavelength around the sphere's circumference. ${ }^{18}$ For $D=345 \mu \mathrm{m}$ and $\lambda=670 \mathrm{~nm}, \mathcal{F}=2.3 \times 10^{6}$, which is to our knowledge the highest value yet reported for an optical interferometer, whereas for $D=680 \mu \mathrm{m}$ and $\lambda=850 \mathrm{~nm}, \mathcal{F}=2.2 \times 10^{6}$ (exceeding the value $\mathcal{F}=1.9 \times 10^{6}$ at $850 \mathrm{~nm}$ found in Ref. 19).

In conclusion, we have recorded quality factors $Q \sim$ $8 \times 10^{9}$ that match that obtained in Ref. 11 for three different wavelengths in the visible and NIR spectra. From the wavelength dependence of the $Q$, it appears as though some mechanism other than absorption in bulk silica limits $Q$ in the NIR. Our data and simple analysis suggest that surface scattering and absorption by adsorbed water could both be responsible.

This study was supported by the U.S. Office of Naval Research (grant N00014-96-1-0580), the National Science Foundation, and the Defense Advanced Research Projects Agency through the Quantum Information and Scholarship Institute administered by the U.S. Army Research Office. D. W. Vernooy acknowledges receipt of a National Science and Engineering Research Council '67 Science and Engineering Scholarship. E. W. Streed acknowledges support from a California Institute of Technology (Caltech) Science and Engineering Research Fellowship. We thank R. Rossi and the Molecular Materials Science Resource Center at Caltech's Beckman Institute for AFM use and expertise and M. L. Gorodetsky for discussion.

*Permanent address, Department of Physics, Moscow State University, Moscow 119899, Russia.

\section{References}

1. G. Griffel, A. Serpengüzel, and S. Arnold, 1995 IEEE Symposium on Frequency Control (Institute of Electrical and Electronics Engineers, New York, 1995).

2. A. Serpengüzel, S. Arnold, and G. Griffel, Opt. Lett. 20, 654 (1995).

3. H. J. Kimble, in Cavity Quantum Electrodynamics, P. R. Berman, ed., Supplement 2 of Advances in Atomic, Molecular and Optical Physics (Academic, San Diego, Calif., 1994), pp. 203-266.

4. H. Mabuchi, Q. A. Turchette, M. S. Chapman, and H. J. Kimble, Opt. Lett. 21, 1393 (1996).

5. V. B. Braginsky and V. S. Ilchenko, Sov. Phys. Dokl. 32, 307 (1987).

6. H. Mabuchi and H. J. Kimble, Opt. Lett. 19, 749 (1994).

7. F. Treussart, J. Hare, L. Collot, V. Lefèvre, D. S. Weiss, V. Sandoghdar, J. M. Raimond, and S. Haroche, Opt. Lett. 19, 1651 (1994).

8. V. Sandoghdar, F. Treussart, J. Hare, V. LefèvreSeguin, J. M. Raimond, and S. Maroche, Phys. Rev. A 54, R1777 (1996).

9. V. B. Braginsky, M. L. Gorodetsky, and V. S. Ilchenko, Phys. Lett. A 137, 393 (1992).

10. L. Collot, V. Lefèvre-Seguin, M. Brune, J.-M. Raimond, and S. Haroche, Europhys. Lett. 23, 327 (1993).

11. M. L. Gorodetsky, A. A. Savchenkov, and V. S. Ilchenko, Opt. Lett. 21, 453 (1995).

12. M. L. Gorodetsky and V. S. Ilchenko, Laser Phys. 2, 1004 (1992).

13. M. L. Gorodetsky and V. S. Ilchenko, Opt. Commun. 113, 133 (1994).

14. D. S. Weiss, V. Sandoghdar, J. Hare, V. Lefèvre-Seguin, J.-M. Raimond, and S. Haroche, Opt. Lett. 20, 1835 (1995).

15. C. Lin, in Handbook of Microwave and Optical Components, K. Chang, ed. (Wiley, New York, 1991), p. 11.

16. H. G. Unger, Planar Optical Waveguides and Fibres (Clarendon, Oxford, 1977), p. 130.

17. G. M. Hale and M. R. Querry, Appl. Opt. 12, 555 (1973).

18. S. C. Hill and R. E. Benner, in Optical Effects Associated with Small Particles, P. W. Barber and R. K. Chang, eds. (World Scientific, Singapore, 1988), pp. 3-61.

19. G. Rempe, R. J. Thompson, H. J. Kimble, and R. Lalezari, Opt. Lett. 17, 363 (1992). 